\title{
Chapter 9 Maintaining compliance with the applicable requirements - Safety Reviews
}

\subsection{Maintaining compliance with the applicable requirements, managing obsolescence and ageing}

Nuclear facility compliance with applicable requirements must be maintained over time. A specific chapter of the General Operating Rules (GOR) is dedicated to periodic inspection and testing which helps verify compliance. This periodic inspection and testing (defined for safety-important elements ${ }^{237}$ ) is supplemented in preventive maintenance programmes, which help avoid the preventive replacement of certain equipment.

Periodic inspection and testing targets two specific types of potential difficulty for research reactors, due to their service life, which can be very long, to their conditions for operation, which can be discontinuous, and to the high neutron fluxes in some structures. These difficulties are:

237. Hereinafter EIP: as per French Order of 7 February 2012, as amended, setting the general rules relative to basic nuclear installations, an EIP is an Element Important for Protection of the interests mentioned in Article L. 593-1 of the French Environment Code. This element contributes to the prevention of risks and drawbacks associated with public security, health and safety or the protection of nature and the environment. 
- equipment obsolescence: on this topic, research reactor instrumentation and control systems dating from the 1970s - 1980s were fully renovated in the late 1990s, including the implementation of programmed software systems ("SIREX racks");

- the ageing of structures and other equipment (cables, polymer materials, etc.); for example, for "neutron beam outside" reactors, the heavy water vessel and neutron or irradiation channels are systematically inspected, given the level of neutron fluxes they are subject to.

Inspections at all stages in an installation's life are the primary responsibility of the facility operators. Nevertheless, it is worth noting that other stakeholders may occasionally intervene to ensure correct implementation (on-site or factories inspections carried out by the French Nuclear Safety Authority (ASN), or by the Nuclear Pressure Equipment Department (DEP) ${ }^{238}$, etc.) - for building structures, IRSN can propose the scheduling milestones and objectives of these inspections to the French Nuclear Safety Authority, as was the case for the Jules Horowitz reactor.

Non-compliance identified by the facility operator during periodic inspection and testing is subject to the same declaration and processing methods as for nuclear power reactors.

However, safety reviews are the opportunity to conduct a detailed review into compliance every ten years, and particularly on how efficiently the facility operator has processed non-compliance identified over the previous ten years. More detailed investigations can be conducted during these reviews.

\subsection{Safety reviews}

\subsubsection{History and approach}

By nature, full safety of a basic nuclear installation can never be definitively achieved. Improvement must be sought, particularly by drawing on feedback and new knowledge.

Since 1978, "safety reviews" have been performed on French power reactors (GCR system reactors, Chooz A pressurised water reactor). This practice was then extended to different series of pressurised water reactors (900 MWe, 1,300 MWe, 1,450 MWe), with an approach that was gradually structured into (ten-year) safety reviews, as specified below. Research reactors operated in France were also subject to safety reviews in the early 1980s, which initially targeted specific subjects. However, from the late 1990s, the principle of systematic ten-year reviews was adopted, following the same approach used for pressurised water reactors in nuclear power plants.

238. For pressure vessels, only equipment that is nuclear class 1 (N1) as per the "ESPN" Order is inspected by ASN (DEP). Equipment that is nuclear class 2 or 3 is inspected by approved bodies. Part of the reactor coolant system for the Jules Horowitz reactor is nuclear class 1 (N1). 


\section{Regulations and approach}

The obligation for basic nuclear installation operators to periodically review (in practice, every ten years) the safety of a basic nuclear installation dates back to the TSN Act in 2006. The safety review process includes several steps with the following two components:

- a facility "compliance assessment" component,

- a facility safety "reassessment" component.

The compliance assessment consists of comparing the real state of the facility with applicable requirements as per the various texts and documents in force: regulations, safety report, general operating rules, etc.

The purpose of the safety reassessment is to assess the safety of the facility with regard to the most recent French and international safety practices and targets, developments in knowledge and operating feedback from the facility or other nuclear installations in France or overseas.

For French research reactors, a safety review currently includes three steps:

- three years before the safety review is due, the operator draws up and sends ASN a "safety review preparation file", specifying the scope and scale of the planned compliance assessment and safety reassessment, which may only focus on some topics, providing appropriate justifications are supplied. In return, after assessment by IRSN, ASN sends a letter to the facility operator with comments on the review preparation;

- the facility operator then conducts a compliancy assessment, including, in particular, inspections of structures, systems and components, and studies for the safety reassessment of its installation;

- after these inspections and studies, the operator sends ASN a review report, specifying the conclusions of its review and the safety improvements it has planned to implement, with the corresponding schedule. After IRSN assessment of this file, and potentially consultation with the standing groups of experts (primarily the standing group of experts for nuclear reactors [GPR], ASN issues an opinion $^{239}$ on the conditions for continued facility operation, and can set additional requirements, particularly concerning works that need to be carried out within specified deadlines.

For a research reactor, works resulting from a safety review may take two or three years to carry out, or more if large amounts of work are deemed necessary. Safety reviews are major steps in the life of a research reactor and can therefore lead to significant work (earthquake reinforcement for concrete structures, improved fire risk protection [compartmentation], etc.) to continue its operation or integrate new technologies.

The following major topics are usually assessed during safety reviews for French research reactors:

239. ASN also sends its opinion to the French minister responsible for Nuclear Safety. There is no "approved" decision. 
- obsolescence and ageing management,

- suitability of containment (structures, ventilation and filtration systems) in the event of a severe accident affecting the reactor or in the event of an external hazard (explosion, plane crash, etc.),

- earthquake resistance, to take into account the most recent seismotectonic data,

- control of fire risks.

In particular, compliance assessments are the opportunity to use in-depth inspections (potentially drilling into concrete structures, etc.) to check that the reactor pool liner and concrete have maintained sufficient characteristics - for reactors designed to resist a potential BORAX-type accident, these characteristics are an essential source of data to ensure conservation of a sufficient water inventory in the pool in such an event.

Similarly, platforms hanging over the reactor pool may need detailed checks on their stability in the event of an earthquake in order to prevent them from falling onto the reactor; the seismic motions considered are generally part of the safety reassessment, in line with new knowledge in this field.

\subsubsection{Examples of significant safety reviews}

As stated above, safety reviews ${ }^{240}$ have regularly been conducted on research reactors since the 1990s. These safety reviews have often focused on a specific safety issue (taking into account operating feedback, significant change to the characteristics or use of the installation, earthquake behaviour reassessment, etc.) and have been an opportunity for in-depth examination of research reactor safety. They have led to the implementation of technical or organisational provisions to improve safety.

This was true for the PHEBUS reactor after the decision was made in the 1980s to conduct experiments which could lead to test fuel meltdown under the Phébus-PF programme. This required continuous operation of the reactor over several weeks (whereas the duration of reactor operation in previous tests had never exceeded a few days per test). These new operating conditions required significant earthquake reinforcement works to ensure that the reactor building could resist a safe shutdown earthquake (it had been designed to resist the maximum historically probable earthquake): building belting, processing of non-compliance discovered during evacuations with regard to the embedding into the rock ( $-5 \mathrm{~m}$ depth) of pillars from auxiliary buildings adjacent to the reactor building.

A leak was detected from the SILOE reactor pool and the decision was made in 1986 to make significant changes (installation of a liner - see section 10.1.2 on this subject) as part of a safety review for this installation.

In the early 1980s, a first review was carried out for the RHF at Grenoble, targeting renovation works on the reactor block and the lifetime of safety-important reactor

240. For the sake of simplicity, the expression "review" will be used in this section, although in some cases, it only corresponds to some of the two-component review content, as defined in the early 1990s and specified above. 
components. Furthermore, following the discovery in 1983 of cracks in the top part of the (internal) concrete containment, at the level of the handling crane bracket, the containment received local external belting in 1989. This operation was especially complicated given the limited space between this containment and the metal containment surrounding it $(80 \mathrm{~cm})$. The prestressing cables had to be inserted through a manhole in the dome of the metal containment.

In the early 1990s, an unusual mark was discovered during a visual examination on an internal RHF structure, the "anti-turbulence grid" ${ }^{241 "}$ located under the core. A second safety review was then conducted for this reactor, with a greater focus on the state of internal reactor structures. In-depth controls showed that there were cracks in one part of this aluminium grid (in AG3NET), which had been directly subject to significant irradiation from the core that had weakened it - this damage had probably been increased by a vibration phenomenon. Given the estimated two-year period required to remove all internal reactor structures and repair or replace the grid, the Laue-Langevin Institute decided after one year to replace the entire reactor block, including the piping sections located in the pool. The facility operator adopted a new design for the "anti-turbulence grid", known as the turned down grid (figure 9.1) that could be replaced more easily. This new grid was qualified by tests conducted in water. These significant works were completed in 1994. The new turned down grid has since only been replaced once.

In 2002, a second safety review of the RHF was conducted, mainly focusing on:

- updating facility safety documents (safety report, general operating rules and on-site emergency plan),

- taking into account lessons learned from facility operation since the previous safety review,

- earthquake behaviour of the facility.

With regard to this last point, the RHF had initially been designed by applying PS 67 earthquake protection rules, considering an earthquake at level 8 on the Mercalli intensity scale ${ }^{242}$, with maximum horizontal ground acceleration of $0.3 \mathrm{~g}$ at basemat le$\mathrm{vel}^{243}$. This seismic reassessment led to significant reinforcements and merits further development.

241. This grid was composed of two layered grids and served to restrict flows entering the core, with water arriving at a (high) speed of $17 \mathrm{~m} / \mathrm{s}$. The fluence (integrated neutron flow over time) to which it had been subject since the start of reactor operation in 1971 was estimated at $3 \times 10^{23}$ neutrons $/ \mathrm{cm}^{2}$.

242. Representing between level VIII and IX on the international macroseismic intensity scale MSK. These are qualitative scales. According to the MSK scale, which became more widely used, level VIII corresponds to "partial collapse of buildings" (conventional) and Level IX to "substantial damage to buildings". The maximum level XII has been defined by "landscape generally changed".

243. Data from Report DSN 50 in 1974. As stated in footnote 198, an acceleration of $0.3 \mathrm{~g}$ corresponds to an area with average earthquake activity. In very active areas (Japan, Turkey, etc.), accelerations can reach 0.4 to $0.6 \mathrm{~g}$. 

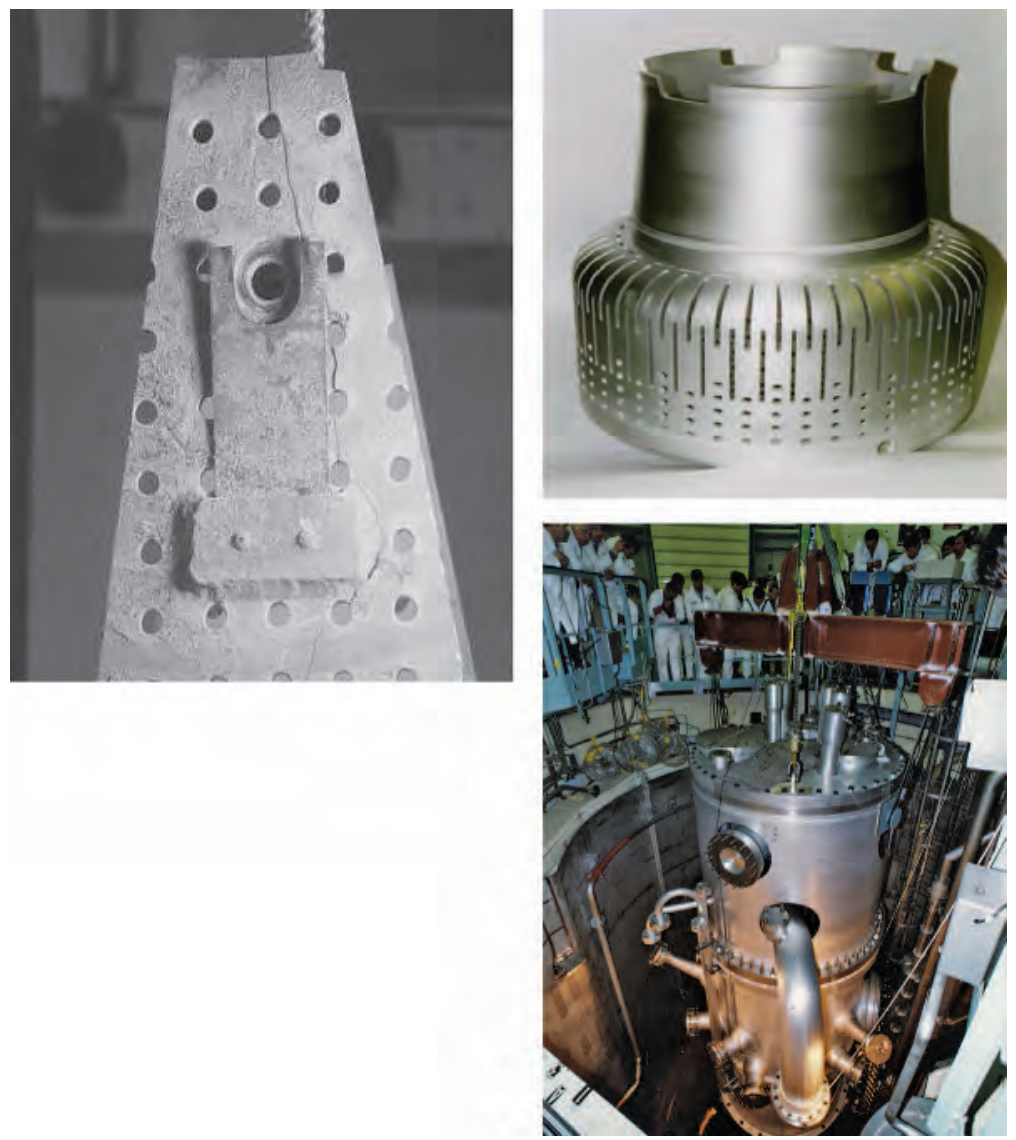

Figure 9.1. Left, the cracked part of the initial "anti-turbulence grid" in the RHF, top-right, the new turned down grid, bottom-right, lowering of the new reactor block into the pool. ( $)$ ILL.

This reassessment was conducted by applying fundamental safety rule (RFS)I.1.c, established in 1992, then, for reinforcement work not yet begun in 2004, the very latest fundamental safety rule 2001-01, with initial assessment ${ }^{244}$ of the site effects (see the focus in section 7.4.2). For the safe shutdown earthquake, application of RFS 2001-01 led to an increase (up to $20 \%$ ) in accelerations for frequencies below $4.5 \mathrm{~Hz}$ - which is particularly important for concrete structures - and a significant decrease in accelerations for the $[4.5 \mathrm{~Hz}-30 \mathrm{~Hz}]$ range. Maximum ground acceleration (or peak ground acceleration - PGA) remained close to $0.3 \mathrm{~g}$.

The operator carried out simulation calculations for the linear behaviour of all structures on the RHF (the calculation mesh is shown in figure 9.2) in order to quantify the resistance deficits of these structures for the new earthquake spectra. The following deficits were identified:

244. The Laue-Langevin Institute partnered with the CASHIMA project, led by the CEA for the part relating to site effects. 

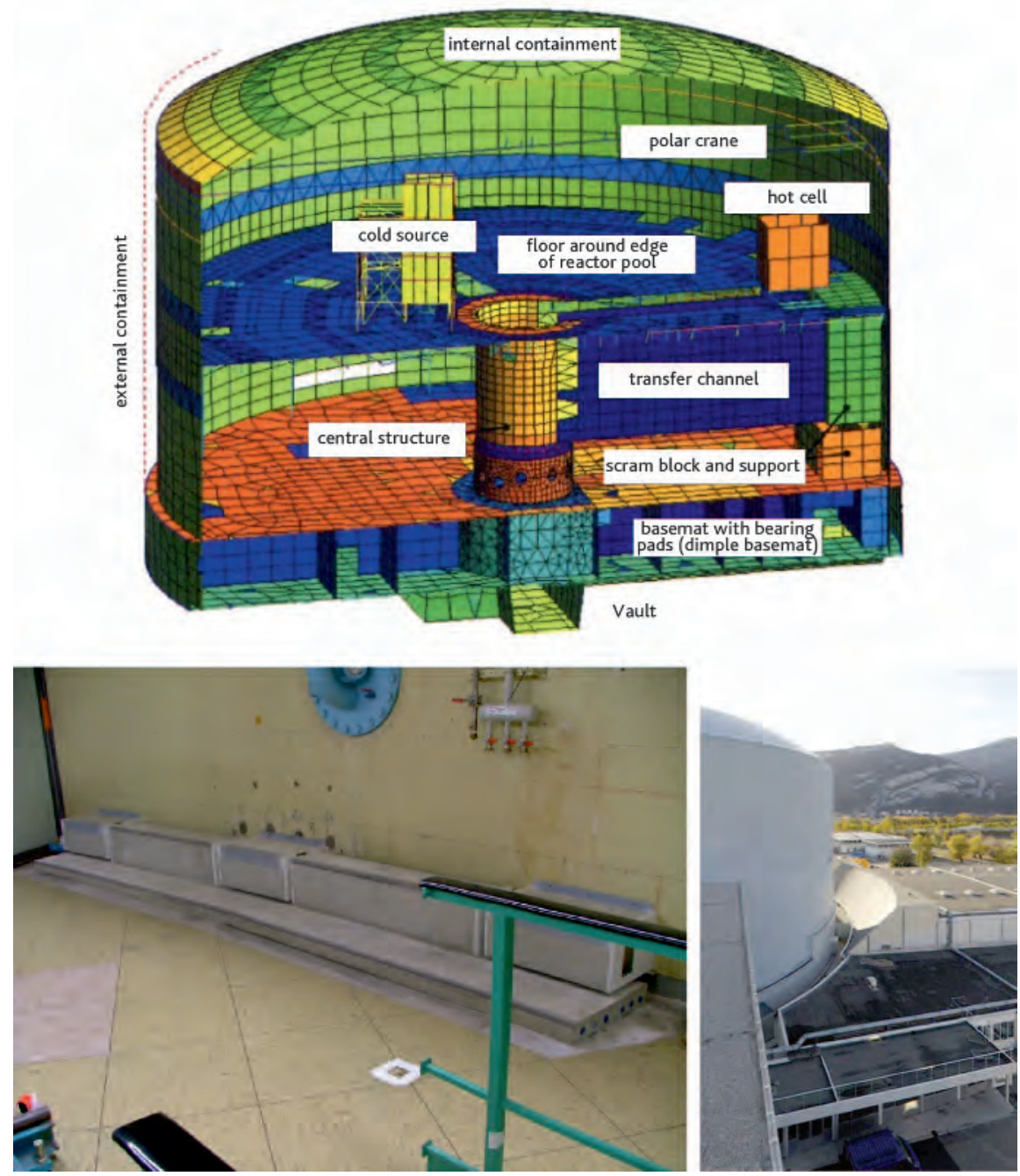

Figure 9.2. Top, reactor concrete structure grid used for earthquake reassessment of the high-flux reactor in Grenoble in the 2000s; bottom-right, view of the part removed from one of the neutron guide halls; bottom-left, partial view of the "teeth" located between the Level D slab and concrete containment. () ILL.

- $15 \%-40 \%$ for the pool's concrete walls,

- $60 \%$ for the extremity of the transfer channel ${ }^{245}$.

These observations led the facility operator to conduct various works to reinforce the reactor:

245. Channel composed of three compartments: Channel 1, Channel 2 and Channel 3, which can be separated using sluice gates (cofferdams). 
- deconstruction of peripheral buildings located on the floor around the edge of the reactor pool (one of which represented a load of 1,500 tonnes);

- creation of a vertical support wall for the transfer channel;

- creation of 70 "teeth" (tangential stops or "comb" system) between the floor around the edge of the reactor pool and the concrete containment wall, with a $1 \mathrm{~mm}$ gap between the teeth (figure 9.2);

- reinforcement of Building ILL4 adjacent to the reactor (building housing the reactor control room) and removal of part of the extremity of this building located closest to the reactor's metal containment in order to create a $20 \mathrm{~cm}$ space between Building ILL4 and the metal containment to prevent impacts between these structures in the event of an earthquake;

- cutting away at $45^{\circ}$ the extremities of the neutron guide halls closest to the metal containment (figure 9.2).

Furthermore, this seismic reassessment of the RHF led to the installation of an emergency core cooling system (CRU) for managing potential leaks from the reactor pool in the event of an earthquake.

The MASURCA reactor was subject to a first safety review in 1988, during which the lessons learned from the first years of reactor operation were analysed. Similarly, the ORPHEE and OSIRIS reactors were subject to safety reviews in 1997 and 1999 respectively. For the ORPHEE reactor, the review mainly focused on provisions for managing accident situations or fire risks. For the OSIRIS reactor, the review conducted in 1999 mainly focused on the lessons learned from operation, fire risk protection and behaviour of the containment in the event of a BORAX-type accident.

The CABRI reactor was also subject to a seismic reassessment as part of an overall safety review integrating plans to install a pressurised water loop. This reassessment led to reinforcements, which were carried out from 2003, mainly consisting of the reinforcement of the walls, columns and beams of the reactor building and auxiliary buildings, and the belting of the reactor building superstructures using reinforced concrete tie beams. 